\title{
Sutureless intrascleral intraocular lens fixation with lamellar dissection of scleral tunnel
}

This article was published in the following Dove Press journal:

Clinical Ophthalmology

27 January 2016

Number of times this article has been viewed

\author{
Takahiro Kawaji ${ }^{1,2}$ \\ Tomoki Sato ${ }^{2}$ \\ Hidenobu Tanihara' \\ 'Department of Ophthalmology, \\ Faculty of Life Sciences, Kumamoto \\ University, Chuo-ku, ${ }^{2}$ Sato Eye \& \\ Internal Medicine Clinic, Kumamoto, \\ Japan
}

Purpose: To report the results of sutureless scleral fixation of a posterior chamber intraocular lens (IOL) by using our developed simple technique.

Methods: We retrospectively reviewed the medical records of 48 eyes of 47 patients who underwent sutureless intrascleral IOL fixation by using our modified technique. A 25-gauge microvitreoretinal knife was used to perform sclerotomies and create limbus-parallel scleral tunnels with lamellar dissection in which the haptics were fixed.

Results: The IOLs were fixed and centered well. The mean follow-up period was 26.7 months. Postoperative complications included smooth vitreous hemorrhage in four eyes (8.3\%), cystoid macular edema in two eyes (4.2\%), and iris capture of the IOL in two eyes (4.2\%). No other complications, such as breakage of the IOL, spontaneous IOL dislocation, or retinal detachment, were detected during the follow-up period.

Conclusion: The lamellar dissection of the limbus-parallel scleral tunnel can simplify the forceps-assisted introduction of the haptics into the scleral tunnel, and this technique seemed to be safe.

Keywords: intraocular lenses, ophthalmologic surgical procedures, intrascleral fixation, sutureless fixation

\section{Introduction}

Intraocular lens (IOL) implantation in an eye without sufficient capsular support has been accomplished by using an anterior chamber IOL, an iris-fixed IOL, and a transscleral-fixed posterior chamber (PC) IOL through the ciliary sulcus or pars plana. ${ }^{1,2}$ Despite the development of these techniques, transscleral IOL fixation is associated with several suture-related complications such as inflammation, suture degradation, delayed IOL dislocation owing to broken sutures, and suture exposureinduced endophthalmitis. ${ }^{3,4}$

Sutureless techniques for intrascleral fixation of a PC IOL have recently become increasingly popular. ${ }^{5-13} \mathrm{We}$ describe here a modified simple intrascleral fixation technique that provides good IOL fixation with reliable wound closure.

\section{Methods}

\section{Subjects and data collection}

We retrospectively reviewed the medical records of patients who had had sutureless intrascleral IOL fixation performed at the Department of Ophthalmology, Kumamoto University Hospital, between April 2012 and May 2013 and who had been followed up for at least 18 months. All patients were informed about the procedure and its possible complications and gave their consent to review their record later. The study was conducted in accordance with the Declaration of Helsinki, and the Institutional
Correspondence: Takahiro Kawaj Department of Ophthalmology, Faculty of Life Sciences, Kumamoto University, I-I-I Honjo, Chuo-ku, Kumamoto 860-8556, Japan

$\mathrm{Tel}+8 \mid 963735247$

Fax +8I 963735249

Email kawag@white.plala.or.jp 
Review Board of Kumamoto University approved this retrospective review and analysis of patient data (reference number: 186).

At all pre- and postoperative visits, all patients had a standard ophthalmologic examination including measurement of best-corrected visual acuity (BCVA) with a Landolt chart, slit-lamp examination, measurement of intraocular pressure (IOP), and dilated indirect slit-lamp biomicroscopy. The density of endothelial cells in the cornea was measured with a specular microscope (EM-3000; TOMEY, Nagoya, Japan) preoperatively and 3 months postoperatively.

\section{Surgical technique}

A sclerocorneal incision of 2.8-3.2 mm was made with the patient under retrobulbar anesthesia, and an infusion cannula or anterior chamber maintainer was inserted. An anterior vitrectomy was performed. A sclerotomy was made $2.0 \mathrm{~mm}$ to the limbus with an angled 25-gauge microvitreoretinal (MVR) knife (Figure 1A [1-3]). The angled MVR knife was used to create the scleral tunnel $3.0-3.5 \mathrm{~mm}$ long and approximately $50 \%$ of the scleral thickness, which was made parallel to the limbus and was started at the sclerotomy (Figure 1B [1-3]). Lamellar scleral dissection, $1.0 \mathrm{~mm}$ long, was then performed at an outer wall of the scleral tunnel (Figure 1C [1-3]). This lamellar dissection of the limbus-parallel scleral tunnel can simplify the forceps-assisted introduction of the haptics into the scleral tunnel. Same sclerotomy, scleral tunnel, and lamellar scleral dissection were made $180^{\circ}$ from the other site. We used a three-piece IOL (NX-70; Santen Pharmaceutical, Tokyo, Japan) with an XJ-70 injector. The IOL was $13.2 \mathrm{~mm}$ long and had a $7 \mathrm{~mm}$ diameter optic, and the haptics were composed of flexible polyvinylidene fluoride. The IOL was inserted into the anterior chamber, and the trailing haptic was kept outside to prevent the IOL from falling into the vitreous cavity. The tip of the haptic was then grasped with a 25-gauge forceps (MAXGrip; Alcon-Grieshaber, Fribourg, Switzerland, or IOL haptic gripping forceps; Geuder, Heidelberg, Germany), pulled through the sclerotomy, and externalized on the left side (Figure 1D). After the trailing haptic was inserted into the anterior chamber with a 25-gauge forceps held in the right hand, the other forceps was used to hold the haptic in the left hand (Figure 1E). Then, the haptic tip was grasped with the forceps, pulled through the sclerotomy, and externalized on the right side (Figure 1F). The tip of the haptic was then inserted into the limbus-parallel scleral tunnel with the forceps, and the IOL was positioned and centered (Figure 1G [1-3]). The conjunctiva was closed with 8-0 polyglactin (surgical technique, Video S1).
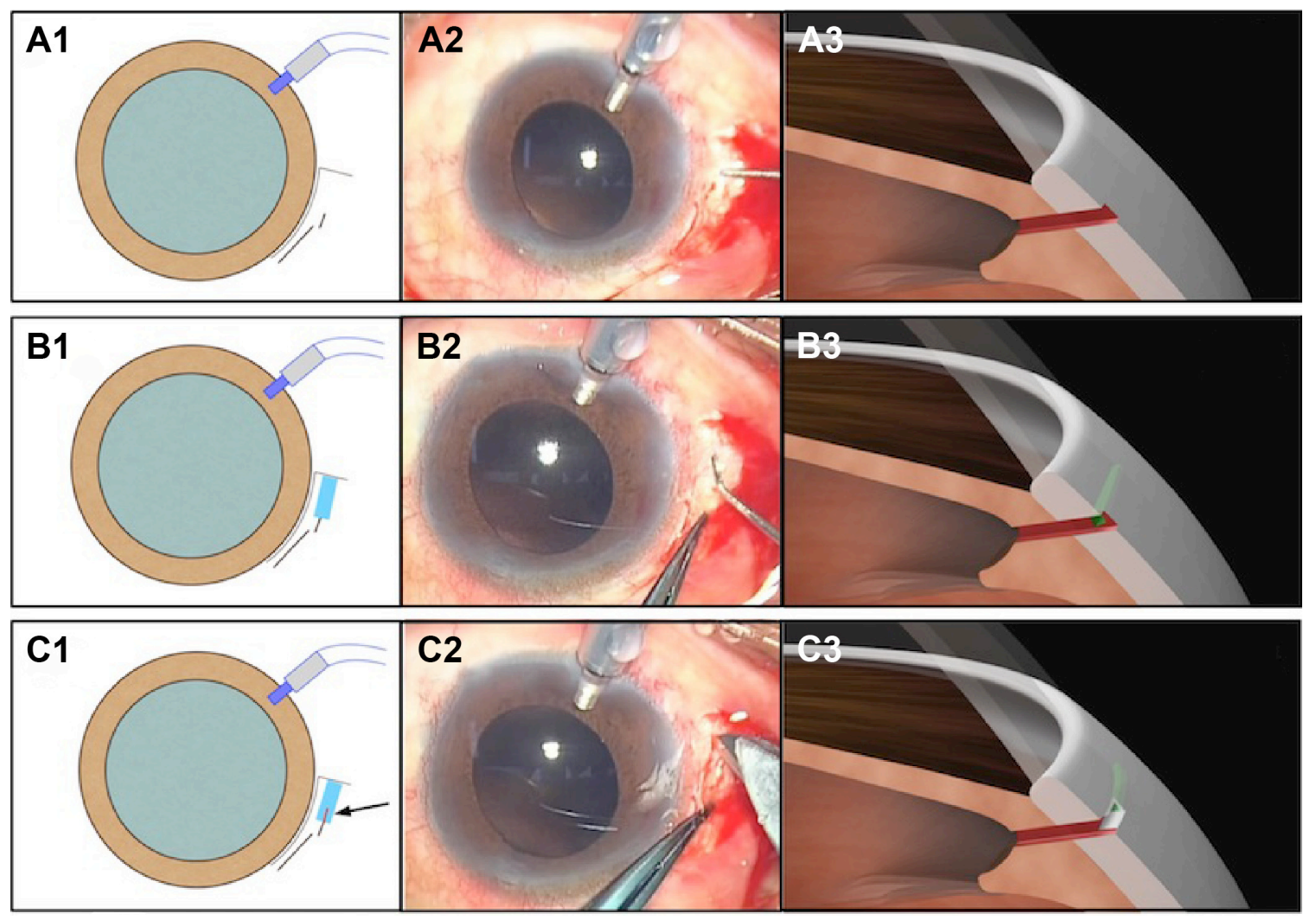

Figure I (Continued) 

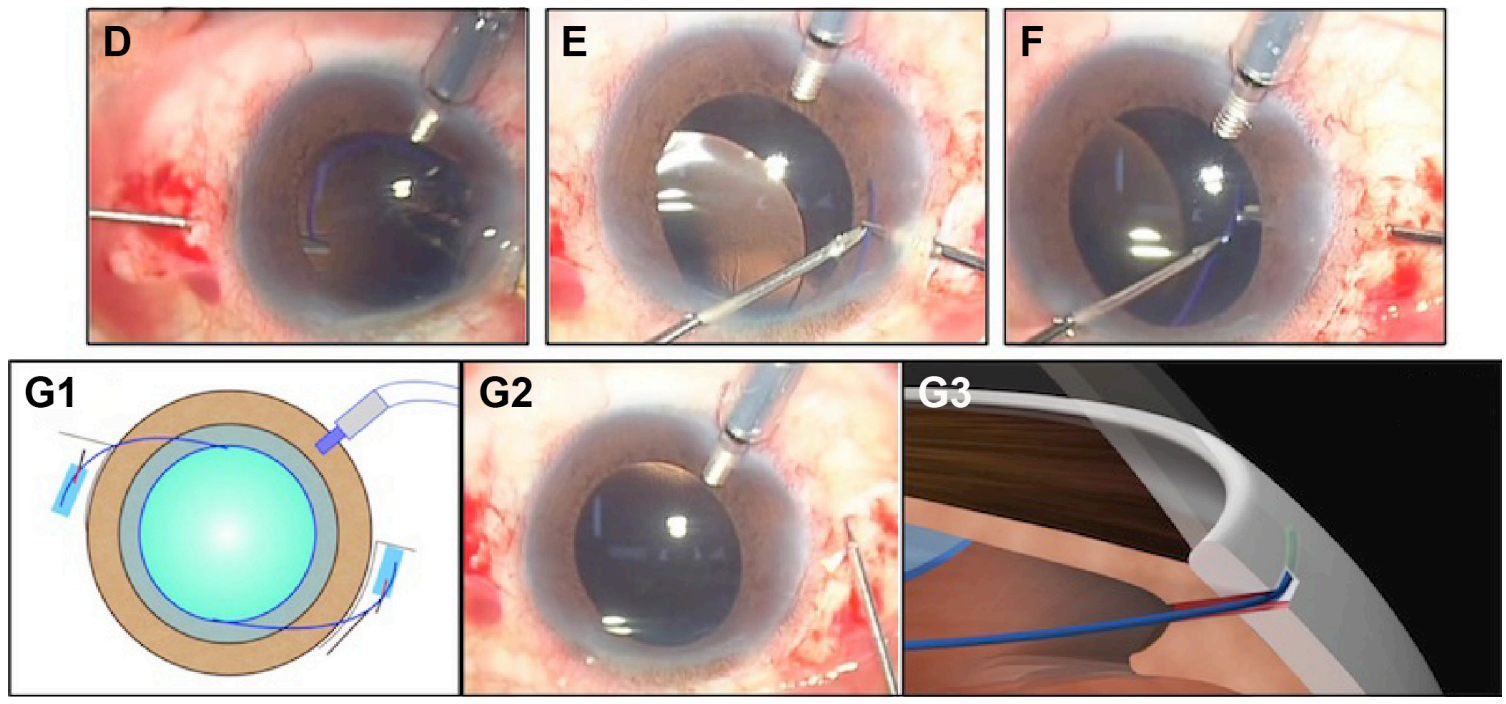

Figure I Surgical technique.

Notes: (A [I-3]) One sclerotomy was made $2.0 \mathrm{~mm}$ parallel to the limbus with a 25-gauge MVR knife. (B [I-3]) One scleral tunnel, 3.0-3.5 mm long and approximately $50 \%$ of the scleral thickness, was made parallel to the limbus by using an angled MVR knife and was started from the sclerotomy site. (C [I-3]) Lamellar scleral dissections, $1.0 \mathrm{~mm}$ long, were then performed at an outer wall of the scleral tunnel (arrow). (D) A three-piece IOL was inserted into the anterior chamber with an injector, and the tip of the haptic was then grasped with a 25-gauge MAXGrip Forceps, pulled through the sclerotomy, and externalized on the left side. (E) After the trailing haptic was inserted into the anterior chamber with a 25-gauge MAXGrip Forceps held in the right hand, the other forceps was used to hold the haptic in the left hand. (F) The haptic tip was grasped with the forceps, pulled through the sclerotomy, and externalized on the right side. (G [I-3]) The tip of the haptic was then inserted into the limbus-parallel scleral tunnel with the forceps, and the IOL was then positioned and centered.

Abbreviations: MVR, microvitreoretinal; IOL, intraocular lens.

\section{Data analysis}

For statistical analyses, the decimal BCVA value was converted to the logarithm of the minimum angle of resolution (logMAR). $P<0.05$ was considered significant. The statistical analyses were performed via JMP, version 9 (SAS Institute Inc., Cary, NC, USA).

\section{Results}

Table 1 shows the baseline characteristics of the 48 eyes of 47 consecutive patients in this study. The mean follow-up period was 26.7 months.

Table 2 shows the summary of surgical outcomes. The mean postoperative BCVA improved significantly at 3 months $(P=0.02)$ and no BCVA value that worsened by at least $0.30 \log$ MAR was seen. There was no significant

Table I Baseline characteristics of the study patients

\begin{tabular}{ll}
\hline Characteristic & Value \\
\hline Number of eyes (number of patients) & $48(47)$ \\
Number of male/female patients & $29 / 18$ \\
Age, years, mean (range) & $70(43-88)$ \\
Preexisting conditions, number of eyes (\%) & \\
PC IOL subluxation or luxation & 2 I (44) \\
Aphakia & II (23) \\
Lens subluxation or luxation & $15(3 \mathrm{I})$ \\
Capsular defect during phacoemulsification & I (2) \\
Follow-up period after surgery, months, mean (range) & $26.7(\mid 8-3$ I) \\
\hline
\end{tabular}

Abbreviations: IOL, intraocular lens; PC, posterior chamber. change in the mean IOP (preoperative IOP, $11.6 \pm 2.4 \mathrm{mmHg}$; postoperative IOP, $12.3 \pm 2.1 \mathrm{mmHg}$ ). Two eyes $(4.2 \%)$ demonstrated an absolute prediction error of more than $1.5 \mathrm{D}$. No other complications, such as iatrogenic distortion or breakage of the IOL haptic, spontaneous IOL decentration that caused the refractive change, endophthalmitis, or retinal detachment, were detected during the follow-up period.

In addition, the IOL haptics were completely incarcerated in the scleral tunnel, and different views of the haptics can be seen, depending on the depth of the tunnel (Figure 2).

Table 2 Summary of surgical outcomes

\begin{tabular}{ll}
\hline Characteristic & Value \\
\hline $\begin{array}{l}\text { Visual acuity, logMAR, mean (SD) } \\
\quad \text { Preoperative }\end{array}$ & $0.38(0.62)$ \\
$\quad$ Postoperative & $0.08(0.23)$ \\
Endothelial cell count, mean (SD) & \\
$\quad$ Preoperative & $2,263(622)$ \\
$\quad$ Postoperative & $1,980(629)$ \\
Percent change in endothelial cell count, mean (SD) & \\
$\quad$ All cases & $12.5(I 5.7)$ \\
$\quad$ Aphakia cases & $8.5(7.0)$ \\
Postoperative refractive error, diopter, mean (SD) & $0.25(0.79)$ \\
Complications, number of eyes (\%) & \\
$\quad$ Smooth vitreous hemorrhage & $4(8.3)$ \\
Cystoid macular edema & $2(4.2)$ \\
Iris capture of the IOL & $2(4.2)$ \\
\hline
\end{tabular}

Abbreviations: IOL, intraocular lens; logMAR, logarithm of the minimum angle of resolution; SD, standard deviation. 

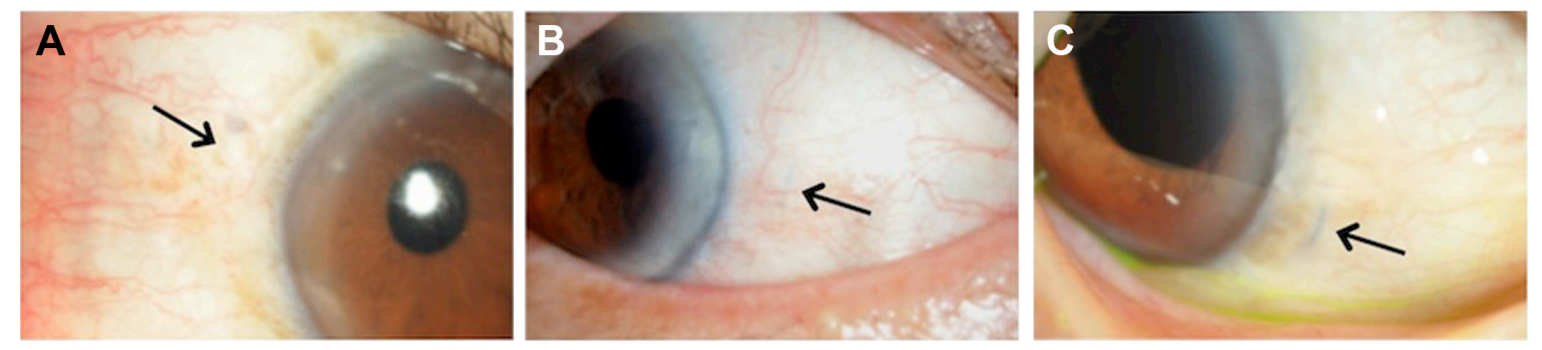

Figure 2 (A-C) One-year postoperative slit-lamp images of the limbal position above the scleral tunnel. Note: Different views of the IOL haptics (arrow) can be seen, depending on the depth of the tunnel.

No conjunctival erosion or inflammation was observed at the site of the IOL haptic.

\section{Discussion}

Several investigators have reported on the techniques for sutureless intrascleral fixation of PC IOLs. ${ }^{5-12}$ These techniques have some advantages over transscleral suturing, which can cause suture-induced inflammation, IOL tilt related to inaccurate placement of the fixation sutures, delayed IOL dislocation owing to suture degradation or breakage, and suture exposure-induced endophthalmitis. ${ }^{2}$ Techniques with or without the use of a scleral flap have been reported. The technique without a scleral flap is simpler, takes less time, and requires neither sutures nor fibrin glue. However, inserting the IOL haptic into the scleral tunnel, as described by Gabor and Pavlidis, ${ }^{6}$ is not easy because the sclerotomy and scleral tunnel are so close to each other. Therefore, to resolve this difficulty, we added a lamellar scleral dissection, approximately $1.0 \mathrm{~mm}$ long, at the outer wall of the scleral tunnels. As a result, the haptic can be easily grasped and introduced into the scleral tunnel with the forceps.

In this study, all eyes were followed up for at least 18 months, with the mean follow-up period being 26.7 months. No IOL dislocation or tilt was observed during this follow-up period, which indicated good intermediate-term IOL stability with our technique. Even if IOL decentration occurs after surgery, it can be easily corrected by reopening the incision in the conjunctiva and sclera and adjusting the position of the IOL haptic.

Several new techniques, including a trocar-assisted, ${ }^{11}$ a sutureless 27 -gauge needle-guided, ${ }^{12}$ and a Y-fixation intrascleral IOL implantation technique, ${ }^{13}$ were recently reported. These techniques do not require a large lamellar scleral flap and fibrin glue, and nor does our technique. In addition, our technique may provide minimal invasion to the surrounding tissues without using a trocar, ${ }^{11}$ even small scleral flap, ${ }^{12}$ and relatively difficult needle technique. ${ }^{13}$ Our study has certain limitations, including a small sample size, intermediate follow-up period, and no control group.

\section{Conclusion}

The lamellar dissection of the limbus-parallel scleral tunnel can simplify the forceps-assisted introduction of the haptics into the scleral tunnel and seems to be a safe technique. Longer follow-up is needed to determine the long-term results and safety of our technique.

\section{Acknowledgments}

This work was supported in part by JSPS KAKENHI Grant Numbers 23390403, 23791994. All authors acknowledge Judith B Gandy, Precision Editing, for editing this manuscript.

\section{Author contributions}

TK performed the surgery, study design, data collection, statistical analysis, and drafted the manuscript. TS and HT participated in the design of the study and revised it. All authors read and approved the final manuscript.

\section{Disclosure}

The authors report no conflicts of interest in this work.

\section{References}

1. Zeh WG, Price FW Jr. Iris fixation of posterior chamber intraocular lenses. J Cataract Refract Surg. 2000;26:1028-1034.

2. Por YM, Lavin MJ. Techniques of intraocular lens suspension in the absence of capsular/zonular support. Surv Ophthalmol. 2005;50: 429-462.

3. Teichmann KD, Teichmann IAM. The torque and tilt gamble. J Cataract Refract Surg. 1997;23:413-418.

4. McAllister AS, Hirst LW. Visual outcomes and complications of scleralfixated posterior chamber intraocular lenses. J Cataract Refract Surg. 2011;37:1263-1269.

5. Maggi R, Maggi C. Sutureless scleral fixation of intraocular lenses. J Cataract Refract Surg. 1997;23:1289-1294.

6. Gabor SG, Pavlidis MM. Sutureless intrascleral posterior chamber intraocular lens fixation. J Cataract Refract Surg. 2007;33:1851-1854.

7. Agarwal A, Kumar DA, Jacob S, Baid C, Agarwal A, Srinivasan S. Fibrin glue-assisted sutureless posterior chamber intraocular lens implantation in eyes with deficient posterior capsules. J Cataract Refract Surg. 2008;34:1433-1438.

8. Rodrıguez-Agirretxe I, Acera-Osa A, Ubeda-Erviti M. Needle-guided intrascleral fixation of posterior chamber intraocular lens for aphakia correction. J Cataract Refract Surg. 2009;35:2051-2053. 
9. Scharioth GB, Prasad S, Georgalas I, Tataru C, Pavlidis M. Intermediate results of sutureless intrascleral posterior chamber intraocular lens fixation. J Cataract Refract Surg. 2010;36:254-259.

10. Kumar DA, Agarwal A, Prakash D, Prakash G, Jacob S, Agarwal A. Glued intrascleral fixation of posterior chamber intraocular lens in children. Am J Ophthalmol. 2012;153:594-601.

11. Totan Y, Karadag R. Trocar-assisted sutureless intrascleral posterior chamber foldable intra-ocular lens fixation. Eye. 2012;26:788-791.
12. Yamane S, Inoue M, Arakawa A, Kadonosono K. Sutureless 27-gauge needle-guided intrascleral intraocular lens implantation with lamellar scleral dissection. Ophthalmology. 2014;121:61-66.

13. Ohta T, Toshida H, Murakami A. Simplified and safe method of sutureless intrascleral posterior chamber intraocular lens fixation: Y-fixation technique. J Cataract Refract Surg. 2014;40:2-7.

\section{Publish your work in this journal}

Clinical Ophthalmology is an international, peer-reviewed journal covering all subspecialties within ophthalmology. Key topics include: Optometry; Visual science; Pharmacology and drug therapy in eye diseases; Basic Sciences; Primary and Secondary eye care; Patient Safety and Quality of Care Improvements. This journal is indexed on

Submit your manuscript here: http://www.dovepress.com/clinical-ophthalmology-journal

\section{Dovepress}

PubMed Central and CAS, and is the official journal of The Society of Clinical Ophthalmology (SCO). The manuscript management system is completely online and includes a very quick and fair peer-review system, which is all easy to use. Visit http://www.dovepress.com/ testimonials.php to read real quotes from published authors. 